\title{
Joint Statistics on Cardiac Shape and Fiber Architecture
}

\author{
Hervé Lombaert ${ }^{1}$ and Jean-Marc Peyrat ${ }^{2}$ \\ 1 Centre for Intelligent Machines, McGill University, Montreal \\ ${ }^{2}$ Qatar Robotic Surgery Centre, Doha, Qatar
}

\begin{abstract}
Cardiac fiber architecture plays an important role in electrophysiological and mechanical functions of the heart. Yet, its inter-subject variability and more particularly, its relationship to the shape of the myocardium, is not fully understood. In this paper, we extend the statistical analysis of cardiac fiber architecture beyond its description with a fixed average geometry. We study the co-variation of fiber architecture with either shape or strain-based information by exploring their principal modes of joint variations. We apply our general framework to a dataset of 8 ex vivo canine hearts, and find that strain-based information appears to correlate best with the fiber architecture. Furthermore, compared to current approaches that warp an average atlas to the patient geometry, our preliminary results show that joint statistics improves fiber synthesis from shape by $8.0 \%$, with cases up to $25.9 \%$. Our experiments also reveal evidence on a possible relation between architectural variability and myocardial thickness.
\end{abstract}

\section{Introduction}

Cardiac disease is the leading cause of death throughout the world. The study of heart structures and functions is essential for better diagnostics and treatments. In particular, the cardiac fiber architecture, a complex structure of myofibers organized as laminar sheets [12, plays an important role in electrophysiology, mechanical functions and remodeling processes [7] of the heart. Yet, its structural variability across a population 4 is not fully understood. Current knowledge has in fact been mostly based on histology studies 11. Recent advances in diffusion tensor imaging (DTI) enable better 3D models of the cardiac fiber and laminar architecture [13. However, existing 3D statistical atlases (on ex vivo human [16] and canine [19] DTI) focus on modeling the average diffusion tensor field in an average cardiac geometry and typically ignore the impact of shape variations on the cardiac fiber and laminar architecture. Current fiber models often rely on simplistic mathematical descriptions [21, where fiber directions are assumed to vary linearly across the myocardium wall, or on registering a DTI atlas onto a patient space [23]. Recent work on coupling shape and fiber variability [10 14] has only focused on the fiber orientation in the left ventricle. Notwithstanding the current models, a general framework for characterizing the joint variability of the complete cardiac fiber architecture with explicit or high-order shape information remains yet to be made. In particular, what shape information correlates best 
with the cardiac fiber and laminar architecture, and can their joint shape and fiber variations improve the synthesis of complete fiber structures? This finds direct application, for instance, in detecting architectural discrepancies, which is relevant for studies on hypertrophic hearts, in in vivo DTI, where acquisition is limited, and in electromechanical simulations with refined patient-specific models of the heart.

This paper proposes a novel statistical framework for analyzing the joint variations between general shape and fiber characteristics. Our approach is inspired by the principal geodesic analysis [9] of DTI [25|17] and extends the statistical analysis of DTI beyond the simple use of atlas geometries. More precisely, we exploit principal modes of joint variations of a Riemannian structure 8[18 25 that embeds both shape and fiber information. We explore shape variability [3] using the most recent advances in morphometry, which include DeformationBased Morphometry (DBM), based on the relative positions of structures, and Tensor-Based Morphometry (TBM), based on local structure differences 156. The next section details our general framework and how it may improve synthesis of diffusion tensor fields. The results investigate on the possible relations between the shape of the myocardium and the cardiac fiber architecture within a population of canine hearts. We additionally show evidence on a hypothetical diversity of fiber architecture in thicker myocardial areas.

\section{Method}

To study the correlation between cardiac shape and fiber architecture, we propose to take advantage of the latest advances in morphometry (DBM/TBM) and diffusion tensor analysis, both embedded into a Log-Euclidean framework [2].

Preliminary Atlas Construction - The variability study necessitates a common reference space. Consequently, an anatomical atlas is first built from $N$ hearts with the method described in [16]. A prior rigid registration aligns all hearts in common space. The underlying symmetric Log-Demons [24] produces then the diffeomorphic transformations $\left\{\phi_{1 \ldots N}\right\}$ that register each rigidly aligned heart onto the average heart. These nonrigid deformations are defined in terms of stationary velocity fields, $\phi=\operatorname{Exp}(v)$. The diffusion tensor fields $\left\{\mathbf{D}_{1 \ldots N}\right\}$ are then warped using these deformations onto the atlas space. The Finite Strain strategy [1] is used since it preserves geometric features 19. Additionally, the variations of temperature during acquisition are compensated for normalizing the diffusion tensor matrices with their distributions modes as in [19. Hereafter, D will represent a compensated and reoriented diffusion tensor field. This atlas construction keeps shape and fiber information as independent, avoiding any bias from one to the other in the joint statistical analysis.

\subsection{Shape and Fiber Information}

Shape Information can be expressed via DBM [3] which analyzes the displacement of material. Unfortunately, the space of transformations $\left\{\phi_{1 \ldots N}\right\}$ typically 


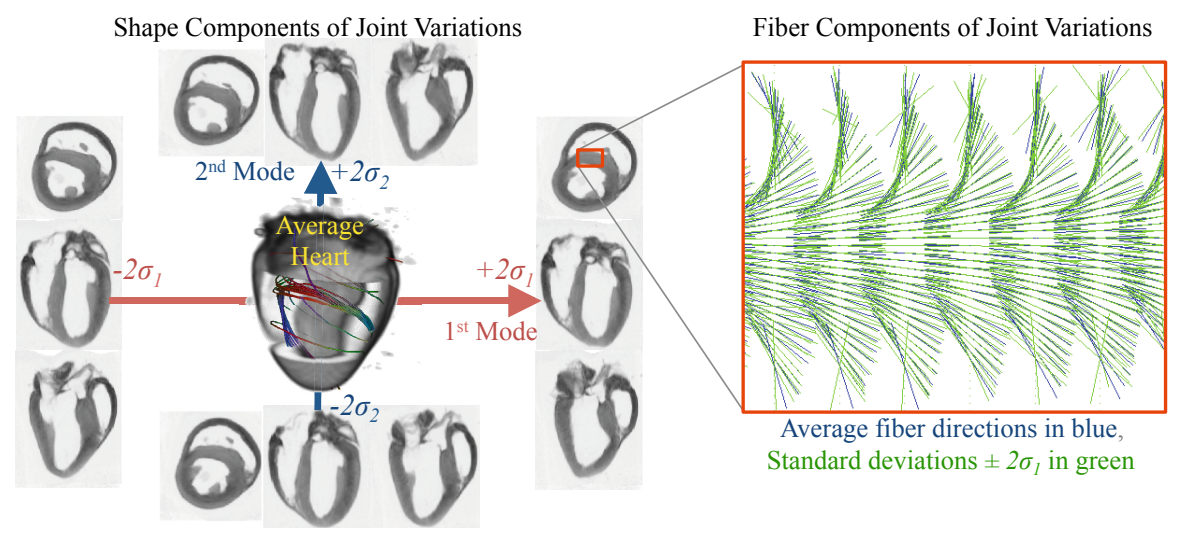

Fig. 1. Average heart warped along two principal modes of joint shape and fiber variations (shown are 3 axial views). Statistics are performed using both velocity components and diffusion tensor information. Shape components, $U^{\text {(shape) }}$, are shown on the left. Fiber components, $U^{\text {(fiber) }}$, in a septal region are shown on the right.

forms a complex Riemannian structure. The velocity fields $\left\{\mathbf{v}_{1 \ldots N}\right\}$ are therefore preferred since they lie on a tangent vector space where standard Euclidean metrics can be used for analysis. Shapes can alternatively be analyzed using TBM, which uses information on local changes in shapes. In particular, the strain tensor measures how a local deformation differs from a rigid transformation, and is therefore adequate for analyzing differences in myocardium walls across a population. Among different definitions, the right Cauchy-Green deformation tensor $C(\phi)=2 E+\mathrm{Id}$, where $E$ is the strain tensor $E=1 / 2\left(\nabla \phi^{T}+\nabla \phi+\nabla \phi^{T} \nabla \phi\right)$, is a $3 \times 3$ symmetric positive semi-definite (SPSD) matrix, and can thus be analyzed in a tangent vector space with its $\log$-representation $\log (C)$, which is also related to the Hencky strain tensor, $\frac{1}{2} \log (C)$. Similarly, $C(\mathbf{v})$, a measure of Riemannian elasticity [5], is also a SPSD matrix and its Logarithm forms a tangent vector space.

Fiber Information consists of the diffusion tensor field $\left\{\mathbf{D}_{1 \ldots N}\right\}$. Each SPSD matrix $D$ can be similarly expressed in the Log-domain with $\log (D)$. Hereafter, we use the compact representation [2] $\operatorname{vec}(D)=\left(D^{x x}, \sqrt{2} D^{x y}, D^{y y}, \sqrt{2} D^{x z}\right.$, $\left.\sqrt{2} D^{y z}, D^{z z}\right)$, which contains the non-repeating elements of $D$ and preserves norms, $\|\operatorname{vec}(D)\|=\|D\|$.

Statistics on shape and fibers are facilitated with previous Log-representations. For instance, averages are computed using the Fréchet mean: [18]: Mean $\left\{\phi_{1 \ldots N}\right\}=$ $\operatorname{Exp}\left(\frac{1}{N} \sum_{i=1}^{N} \mathbf{v}_{i}\right), \operatorname{Mean}\left\{C_{1 \ldots N}\right\}=\operatorname{Exp}\left(\frac{1}{N} \sum_{i=1}^{N} \log \left(C_{i}\right)\right)$, and $\operatorname{Mean}\left\{D_{1 \ldots N}\right\}=$ $\operatorname{Exp}\left(\frac{1}{N} \sum_{i=1}^{N} \log \left(D_{i}\right)\right)$. 


\subsection{Statistics on Joint Shape and Fiber Variations}

Rather than considering changes in shape and in fiber characteristics as individual features, we propose to study their joint variations, which describes how each influence one another.

Joint Data - Let us first define the vector $x^{\text {(shape) }}$ as the raster-scan of shape information across a region $\Omega$ of the heart with $n=|\Omega|$ voxels, with, for instance, the velocity components within $\Omega, x^{\text {(shape })}=\left(v_{1}^{x, y, z}, \ldots, v_{n}^{x, y, z}\right)^{T}$, or the compact deformation Log-tensors, $x^{(\text {shape })}=\left(\operatorname{vec}\left(\log \left(C_{1}\right)\right), \ldots, \operatorname{vec}\left(\log \left(C_{n}\right)\right)\right)^{T}$. Similarly, let us define the vector $x^{(\text {fiber })}=\left(\operatorname{vec}\left(\log \left(D_{1}\right)\right), \ldots, \operatorname{vec}\left(\log \left(D_{n}\right)\right)\right)^{T}$.

The joint data matrix is the concatenation $X=\left(\begin{array}{c}\mathbf{x}^{\text {(shape) }} \\ \mathbf{x}^{\text {(fiber })}\end{array}\right)-\left(\begin{array}{c}\mu^{\text {(shape) }} \\ \mu^{\text {(fiber) }}\end{array}\right)$, with $N$ columns, each with the shape and fiber information of one heart, centered around their average values $\mu^{(\cdot)}=\frac{1}{N} \sum_{j=1}^{N} x_{j}^{(\cdot)}$.

Principal Modes of Joint Variations - We now find the orthonormal basis $U=\left\{u_{1 \ldots N-1}\right\}$ that maximizes the variability within the data $X: u_{1}=$ $\operatorname{argmax}_{\|u\|=1} \sum_{i=1}^{N}\left\langle u, x_{i}\right\rangle^{2}$ and $u_{k=1 \ldots N-1}=\operatorname{argmax}_{\|u\|=1} \sum_{i=1}^{N} \sum_{j=1}^{k-1}\left\langle u_{j}, x_{i}\right\rangle^{2}+$ $\left\langle u, x_{i}\right\rangle^{2}$.

Since $X$ lies on a Euclidean vector space, the inner product is set with $\langle u, x\rangle=u^{T} x[18$, and the principal components $U$ are computed with the eigen decomposition of the covariance matrix $X X^{T}=U \Lambda U^{T}$. Fortunately, the $N-1$ non-trivial eigenvectors $U$ of $X X^{T}$ can be efficiently computed [22] by decomposing the smaller $N \times N$ Gram matrix $X^{T} X=V \Lambda V^{T}$, with $U=X V$. Each eigenvector $u_{j}$, a column of $U$, is a principal mode of joint variation (illustrated in Fig. 1), and can be separated into shape and fiber components since $u_{j}=\left(u_{j}^{\text {(shape) }}, u_{j}^{(\text {fiber })}\right)$, e.g., $u_{j}^{(\text {fiber })}$ is the last $6|\Omega|$ elements of vector $u_{j}$. Note that any type of shape and fiber information lying on vector spaces may be used, for instance, Fig. 1 illustrates such joint variation modes when shape is modeled with velocity field components.

\subsection{Joint Shape and Fiber Space}

Spectral Coordinates - The projection of $x$ onto the principal modes of variations $U$ provides a compact representation of a heart with the spectral coordinate $p=(x-\mu)^{T} U$ in $N-1$ dimensions, i.e., it is the heart coordinate in the joint shape and fiber space (illustrated in Fig. (2). Reciprocally, a spectral coordinate $p$ can synthesize data, $\hat{x}=\mu_{x}+U p^{T}$, for instance, the diffusion tensor field $D_{p}$ :

$$
D_{p}=\operatorname{Exp}\left(\mu^{(\text {fiber })}+\sum_{i=1}^{K} p_{i} u_{i}^{(\text {fiber })}\right),
$$

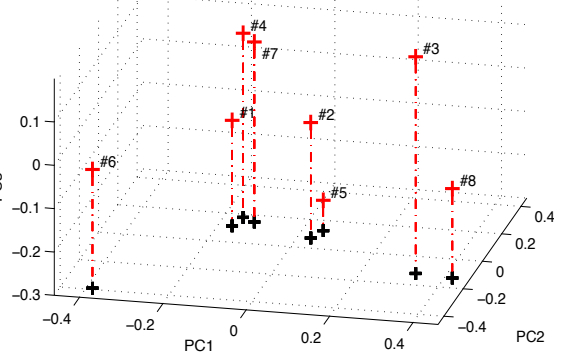

Fig. 2. Joint shape and fiber space where points represent hearts 

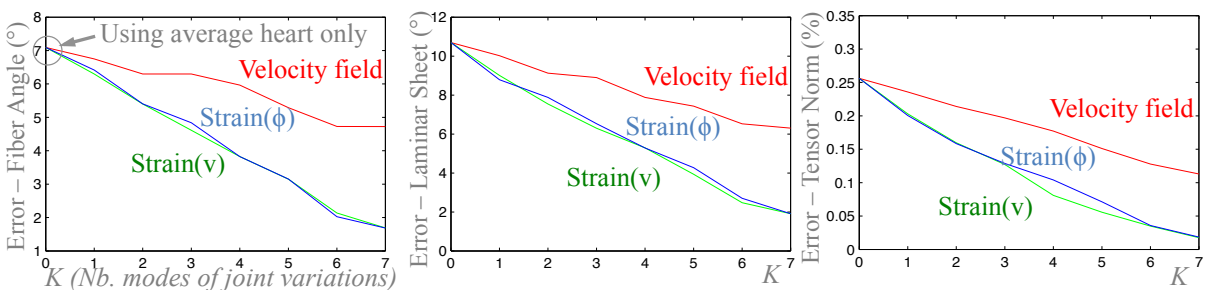

a) Error on Fiber Reconstruction

b) Error on Laminar Sheet Reconstruction

c) Error on DTI Reconstruction

Fig. 3. Error when reconstructing from shape with an increasing number of variation modes. a) Fiber angular differences with ground truth ( $\varepsilon_{e_{1}}$ in degrees). $b$ ) Angular error of the laminar sheet normal $\left(\varepsilon_{e_{3}}\right)$. c) Norm differences of DTI Log-tensor $\left(\varepsilon_{D}\right)$. Strain-based measures (green/blue curves) appears to better capture the joint variability between shape and fiber architecture (lower is better).

with $K=N-1$. If $u^{\text {(shape) }}$ is expressed in terms of a velocity field, the heart shape can also be synthesized with $\phi_{p}=\operatorname{Exp}\left(\mu^{(\text {(shape })}+\sum_{i=1}^{K} p_{i} u_{i}^{\text {(shape) }}\right)$.

Partial Spectral Projection - Partial information may be similarly used to infer the spectral coordinate $\hat{p}$ of a heart. For instance, if only shape information is available for a heart, the projection of $x^{\text {(shape) }}$ onto the normalized eigenvectors $U^{\text {(shape) }}$ approximates $\hat{p}=\left(x^{\text {(shape) }}-\mu_{x}\right)^{T} U^{\text {(shape) }}$. The whole shape and fiber information, $\hat{x}=\left(\hat{x}^{\text {(shape) }} ; \hat{x}^{\text {(fiber) }}\right)$, is in turn recovered using the spectral reconstruction (Eq. (1)).

\section{Results}

We apply the proposed joint statistical framework to the JHU dataset of 8 ex vivo canine hearts [12 (DTI with $b=0$ images of size $256 \times 256 \times 130$, resolution $0.31 \times 0.31 \times 0.80)$ in order 1 ) to determine which shape information (DBM or TBM) has the best correlation with fiber architecture, 2) to show the advantage of using second-order joint statistics over a first-order average atlas to synthesize fibers in a given geometry, and 3) to propose some insights on shape-fiber correlations.

\subsection{Correlation between Cardiac Shape and Fiber Architecture}

We now verify how much information is correlated between cardiac shape and fiber architecture. To do so, we utilize the principal modes of variations of the joint data matrix in order to reconstruct the diffusion tensor field Eq. (1) from different types of shape characteristics: a) from explicit velocity components, $\left.x^{(\text {shape })}=\left(v_{1}, \ldots, v_{n}\right)^{T}, \mathrm{~b}\right)$ Riemannian elasticity, $x^{(\text {shape })}=\left(\operatorname{vec}\left(\log \left(C\left(\mathbf{v}_{1}\right)\right)\right), \ldots\right.$, $\left.\operatorname{vec}\left(\log \left(C\left(\mathbf{v}_{n}\right)\right)\right)\right)^{T}$, and c) Cauchy-Green strain, $x^{(\text {shape })}=\left(\operatorname{vec}\left(\log \left(C\left(\phi_{1}\right)\right)\right), \ldots\right.$, $\left.\operatorname{vec}\left(\log \left(C\left(\phi_{n}\right)\right)\right)\right)^{T}$. The explicit use of velocity fields (DBM) has the advantage to produce synthetic transformations, i.e., geometries can be synthesized. Fig. 1 

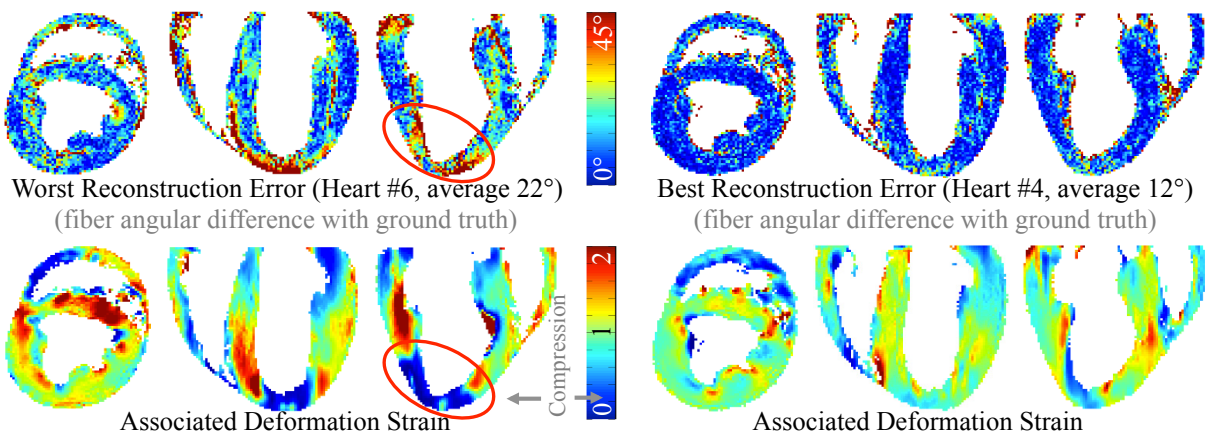

Best Reconstruction Error (Heart \#4, average $12^{\circ}$ ) (fiber angular difference with ground truth)

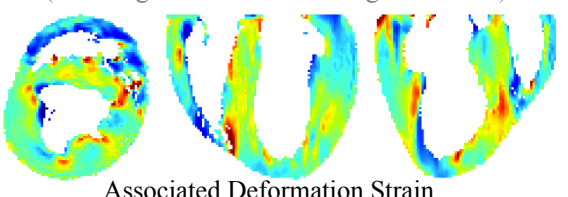

Fig. 4. Reconstruction error showing the angular difference (in degrees) between reconstructed fibers and ground truth. Worst case on left, best on right. The deformation strain suggests that larger errors may occur in areas with high compression in blue (i.e., in thicker myocardial regions).

illustrates such synthesized hearts with spectral coordinates varying between plus or minus two standard deviations, $p_{i}= \pm 2 \sqrt{\lambda_{i}}$. However, strain-based models (TBM) capture shape information on a higher order level that describes local nonrigid morphological changes. We study these two approaches by comparing their reconstruction capabilities.

The diffusion tensor fields of all hearts are reconstructed from spectral coordinates (using Eq. (1)) that are approximated with our three types of shape information. An increasing number of modes of joint variations is used during reconstruction, from $K=0$, which corresponds to what the current state-of-theart is capable of, i.e., reusing only the average tensor field, to $K=N-1$, which takes advantage of the full joint variations between shape and fiber architecture.

The reconstruction error $\varepsilon_{\hat{D}}$ is defined as a geodesic distance between the true and the reconstructed tensor field, $\mathbf{D}$ from the dataset and $\hat{\mathbf{D}}$ from Eq. (1): $: \varepsilon_{\hat{D}}=$ $\frac{1}{|\Omega|} \sum_{i \in \Omega}\left\|\log \left(D_{i}\right)-\log \left(\hat{D}_{i}\right)\right\|^{2}$ where $\Omega$ covers the myocardium. Additionally, the angular difference of fiber orientations between the true fiber field (defined with the first eigenvector $e_{1}$ of $\left.D\right)$ and the reconstructed fiber field $\left(\hat{e}_{1}\right.$ of $\left.\hat{D}\right)$ is measured with $\varepsilon_{\hat{e}_{1}}=\frac{1}{|\Omega|} \sum_{i \in \Omega}^{n} \cos ^{-1}\left|e_{1}(i) \cdot \hat{e}_{1}(i)\right|$. The angular difference of the third eigenvector of $D, \varepsilon_{\hat{e}_{3}}$, quantifies in a similar manner the reconstruction error of the laminar sheet structure 12 . Fig. 3] shows a decreasing reconstruction error when more modes of joint variations are used.

This experiment shows that fiber architecture is correlated with the shape of the heart. Furthermore, strain-based information (TBM) appears to better capture the joint variability between shape and fiber architecture in comparison to using explicit deformation components (DBM).

\subsection{Reconstruction of Cardiac Fiber Architecture from Shape}

Our joint statistical framework is now evaluated with a leave-one-out strategy, where $N$ atlases are built and analyzed by successively removing one heart at a time from the training set. The diffusion tensor fields of omitted hearts are recon- 
Table 1. Leave-one-out - Improvements in tensor reconstruction

\begin{tabular}{|c||c|c|c|c|c|c|c|c||c|c|}
\hline Heart & $\# 1$ & $\# 2$ & $\# 3$ & $\# 4$ & $\# 5$ & $\# 6$ & $\# 7$ & $\# 8$ & $\begin{array}{c}\text { Total } \\
\text { Improv. } \epsilon_{\hat{D}}\end{array}$ & $\begin{array}{c}\text { Total } \\
\text { Improv. } \sigma_{\epsilon_{\hat{D}}}\end{array}$ \\
\hline Velocity & $-0.8 \%$ & $-0.8 \%$ & $5.6 \%$ & $\mathbf{1 6 . 4 \%}$ & $1.6 \%$ & $-2.4 \%$ & $\mathbf{1 5 . 0 \%}$ & $5.4 \%$ & $\mathbf{5 . 0 \%}$ & $3.0 \%$ \\
\hline Strain $(\mathrm{v})$ & $-0.2 \%$ & $-1.1 \%$ & $9.0 \%$ & $\mathbf{2 5 . 8 \%}$ & $0.8 \%$ & $-0.8 \%$ & $\mathbf{1 9 . 5 \%}$ & $11.1 \%$ & $\mathbf{8 . 0 \%}$ & $4.1 \%$ \\
\hline Strain $(\phi)$ & $-0.3 \%$ & $0.4 \%$ & $6.8 \%$ & $\mathbf{2 5 . 9 \%}$ & $0.6 \%$ & $-0.5 \%$ & $\mathbf{1 9 . 3 \%}$ & $9.3 \%$ & $\mathbf{7 . 7 \%}$ & $3.6 \%$ \\
\hline
\end{tabular}
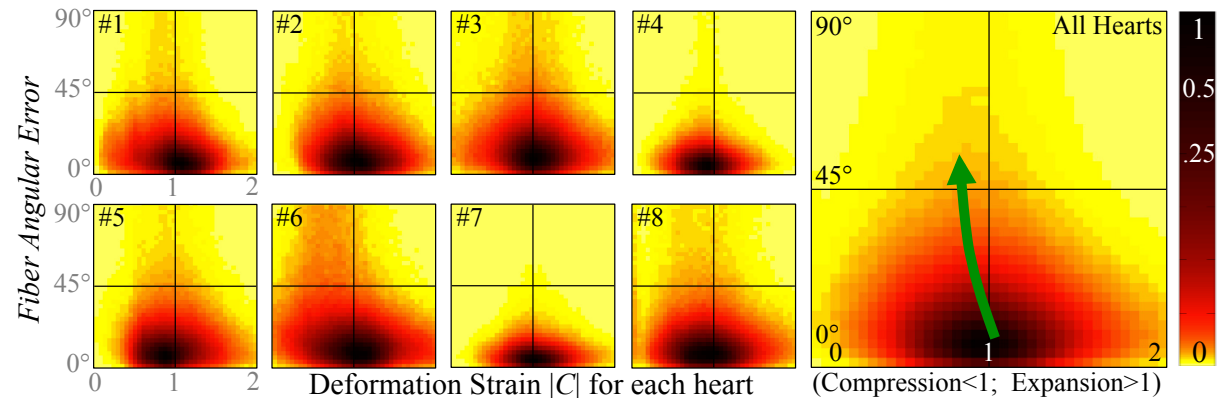

Fig. 5. Joint distribution of reconstruction error, $\varepsilon_{\hat{e}_{1}}$ on the $y$-axis (fiber angular error), and deformation, $|C|$ on the $x$-axis (strain determinant) for each and all hearts. Coloring is the joint probability in a log-scale. Larger reconstruction errors $\left(\varepsilon_{\hat{e}_{1}}>45^{\circ}\right)$ are more frequent in compressed areas (mode at $|C|=0.88$, illustrated with the green arrow), i.e., shape and fiber directions may be less correlated in thicker myocardial regions.

structed, as described earlier, and we measure the improvement in reconstruction accuracy when compared to registering a DTI atlas. Warping an average tensor field is considered here as the current state-of-the-art for synthesizing fibers [23]. We compare $\varepsilon_{\hat{D}}$ with the reconstruction error of a warped atlas, $\varepsilon_{\mu_{D}}$. Table 1 shows the improvement for each heart, which is measured with $\left(\varepsilon_{\hat{D}}-\varepsilon_{\mu_{D}}\right) / \varepsilon_{\mu_{D}}$. We also measure the decrease in the standard deviation of reconstruction error, $\sigma_{\varepsilon_{\hat{D}}}$. The reconstruction improves by $5.0 \%$ for $\varepsilon_{\hat{D}}$, and $3.0 \%$ for $\sigma_{\varepsilon_{\hat{D}}}$, when using velocity components as shape information; $8.0 \%$ and $4.1 \%$ for $\varepsilon_{\hat{D}}$ and $\sigma_{\varepsilon_{\hat{D}}}$ when using the Riemannian elasticity Log-tensor, and $7.7 \%$ and $3.6 \%$ for $\varepsilon_{\hat{D}}$ and $\sigma_{\varepsilon_{\hat{D}}}$ when using the deformation strain Log-tensor. Fig. 4 shows the worst and best reconstruction when using the deformation strain Log-tensor, with respectively $-0.5 \%$ and $25.9 \%$ improvements on $\varepsilon_{\hat{D}} ;-0.4 \%$ and $15.8 \%$ on fiber angular errors $\varepsilon_{\hat{e}_{1}}$; and $-1.1 \%$ and $13.9 \%$ on angular errors of the laminar sheet normal $\varepsilon_{\hat{e}_{3}}$. Note that areas with larger discrepancies appears to be in areas subject to compression, i.e., in thicker myocardial areas.

\subsection{Localization of Higher Reconstruction Error}

The joint probability between the reconstruction error and the deformation strain determinant is studied in order to verify the last observation, notably in thicker myocardial areas. Fig. 5 shows such 2D histograms for all hearts. Areas with high angular errors, $\varepsilon_{\hat{e}_{1}}>45^{\circ}$, have in fact an average distribution mode 
inferior to 1 , at $|C|=0.88$, which suggests, perhaps, that higher reconstruction errors tend to happen in areas subject to compression. This may indicate that cardiac shape and fiber information are less correlated in thicker myocardial regions.

\section{Conclusion}

We presented a general statistical framework where both shape and fiber information are exploited concurrently. Our method extends previous work on joint statistics [1014, which is only based on fiber orientation in the left ventricle, to a complete description of fiber and laminar architecture in the whole heart. Moreover, it also extends the description of shape variability using a higher-order description of shape differences with strain tensors (TBM), which appears to be better correlated with the cardiac fiber architecture. Our experiments showed that such joint variability facilitates the synthesis of complete cardiac fiber and laminar architectures. Compared to current approaches that only use an average atlas, our method infers a diffusion tensor field from shape with an improvement of $8.0 \%$ on average, with cases up to $25.9 \%$. The reconstruction accuracy would most probably increase with the availability of new hearts since they would further refine the joint shape and fiber variability. An additional experiment also revealed that shape and fiber information may be less correlated in thicker myocardial regions. This finding may be relevant in the study of hypertrophic hearts. Future work will use high-order fiber models [20] and focus on other fiber structures, such as in muscles and brains.

Acknowledgments The authors would like to thank Tommaso Mansi and Kaleem Siddiqi for helpful comments. Funding is from Fonds de Recherche Québec (FRQNT).

\section{References}

1. Alexander, D.C., Pierpaoli, C., Basser, P.J., Gee, J.C.: Spatial transformations of diffusion tensor magnetic resonance images. TMI 20 (2001)

2. Arsigny, V., Fillard, P., Pennec, X., Ayache, N.: Log-Euclidean metrics for fast and simple calculus on diffusion tensors. MRM 56 (2006)

3. Ashburner, J., Friston, K.: Voxel-Based Morphometry. NeuroImage 11 (2000)

4. Bronzino, J.D.: Biomedical Engineering Handbook. Springer (1995)

5. Brun, C., Lepore, N., Pennec, X., Chou, Y.Y., Lopez, O., Aizenstein, H., Becker, J., Toga, A.W., Thompson, P.: Comparison of standard and riemannian fluid registration for Tensor-Based morphometry in HIV/AIDS. In: MICCAI (2007)

6. Brun, C., Leporé, N., Pennec, X., Lee, A., Barysheva, M., Madsen, S., Avedissian, C., Chou, Y.Y., de Zubicaray, G., McMahon, K., Wright, M., Toga, A.W., Thompson, P.: Mapping the regional influence of genetics on brain structure variability A Tensor-Based morphometry study. NeuroImage 48 (2009)

7. Costa, K., Holmes, J., Mcculloch, A.: Modelling cardiac mechanical properties in three dimensions. Math. Ph. and Eng. Sc. 359 (2001) 
8. Fletcher, T., Joshi, S.: Riemannian geometry for the statistical analysis of diffusion tensor data. Signal Processing 87 (2007)

9. Fletcher, T., Lu, C., Pizer, S., Joshi, S.: Principal geodesic analysis for the study of nonlinear statistics of shape. TMI 23 (2004)

10. Gil, D., Garcia-Barnes, J., Hernández-Sabate, A., Marti, E.: Manifold parametrization of the lv for statistical modelling of its complete anatomy. In: SPIE (2010)

11. Greenbaum, R., Ho, S., Gibson, D., Becker, A., Anderson, R.: Left ventricular fibre architecture in man. British Heart Journal 45 (1981)

12. Helm, P.A., Tseng, H.J., Younes, L., McVeigh, E., Winslow, R.: Ex vivo 3D diffusion tensor imaging and quantification of cardiac laminar structure. MRM (2005)

13. Hsu, E.W., Henriquez, C.S.: Myocardial fiber orientation mapping using reduced encoding diffusion tensor imaging. JCMR 3 (2001)

14. Lekadir, K., Ghafaryasl, B., Muñoz-Moreno, E., Butakoff, C., Hoogendoorn, C., Frangi, A.F.: Predictive modeling of cardiac fiber orientation using the Knutsson mapping. In: Fichtinger, G., Martel, A., Peters, T. (eds.) MICCAI 2011, Part II. LNCS, vol. 6892, pp. 50-57. Springer, Heidelberg (2011)

15. Lepore, N., Brun, C., Chou, Y.Y., Chiang, M., Dutton, R., Hayashi, K., Luders, E., Lopez, O., Aizenstein, H., Toga, A., Becker, J., Thompson, P.: Generalized tensor-based morphometry of HIV using multivariate statistics on tensors. TMI (2008)

16. Lombaert, H., Peyrat, J.M., Croisille, P., Rapacchi, S., Fanton, L., Cheriet, F., Clarysse, P., Magnin, I., Delingette, H., Ayache, N.: Human atlas of the cardiac fiber architecture: Study on a healthy population. TMI 31 (2012)

17. Merchant, S., Jiang, Y., Joshi, S., Hsu, E.: Assessment of variability of the mouse myocardial fiber structure via principal component analysis. In: WorldComp (2012)

18. Pennec, X., Fillard, P., Ayache, N.: A Riemannian framework for tensor computing. IJCV 66(1) (2006)

19. Peyrat, J.M., Sermesant, M., Pennec, X., Delingette, H., Xu, C., McVeigh, E.R., Ayache, N.: A computational framework for the statistical analysis of cardiac diffusion tensors: application to a small database of canine hearts. TMI 26 (2007)

20. Savadjiev, P., Strijkers, G.J., Bakermans, A.J., Piuze, E., Zucker, S.W., Siddiqi, K.: Heart wall myofibers arranged in minimal surfaces to optimize organ function. PNAS (2012)

21. Sermesant, M., Rhode, K., Sanchez-Ortiz, G.I., Camara, O., Andriantsimiavona, R., Hegde, S., Rueckert, D., Lambiase, P., Bucknall, C., Rosenthal, E., Delingette, H., Hill, D.L., Ayache, N., Razavi, R.: Simulation of cardiac pathologies using an electromechanical biventricular model and XMR. MedIA 9 (2005)

22. Turk, M., Pentland, A.: Eigenfaces for recognition. JCN 3 (1991)

23. Vadakkumpadan, F., Arevalo, H., Ceritoglu, C., Miller, M., Trayanova, N.: Imagebased estimation of ventricular fiber orientations for personalized modeling of cardiac electrophysiology. TMI 31 (2012)

24. Vercauteren, T., Pennec, X., Perchant, A., Ayache, N.: Symmetric log-domain diffeomorphic registration: A demons-based approach. In: Metaxas, D., Axel, L., Fichtinger, G., Székely, G. (eds.) MICCAI 2008, Part I. LNCS, vol. 5241, pp. 754761. Springer, Heidelberg (2008)

25. Xie, Y., Vemuri, B.C., Ho, J.: Statistical analysis of tensor fields. In: Jiang, T., Navab, N., Pluim, J.P.W., Viergever, M.A. (eds.) MICCAI 2010, Part I. LNCS, vol. 6361, pp. 682-689. Springer, Heidelberg (2010) 